ARTICLE

\title{
Multi-ancestry GWAS of the electrocardiographic PR interval identifies 202 loci underlying cardiac conduction
}

Ioanna Ntalla et al.

The electrocardiographic PR interval reflects atrioventricular conduction, and is associated with conduction abnormalities, pacemaker implantation, atrial fibrillation (AF), and cardiovascular mortality. Here we report a multi-ancestry $(N=293,051)$ genome-wide association meta-analysis for the PR interval, discovering 202 loci of which 141 have not previously been reported. Variants at identified loci increase the percentage of heritability explained, from $33.5 \%$ to $62.6 \%$. We observe enrichment for cardiac muscle developmental/contractile and cytoskeletal genes, highlighting key regulation processes for atrioventricular conduction. Additionally, 8 loci not previously reported harbor genes underlying inherited arrhythmic syndromes and/or cardiomyopathies suggesting a role for these genes in cardiovascular pathology in the general population. We show that polygenic predisposition to PR interval duration is an endophenotype for cardiovascular disease, including distal conduction disease, $A F$, and atrioventricular pre-excitation. These findings advance our understanding of the polygenic basis of cardiac conduction, and the genetic relationship between PR interval duration and cardiovascular disease. 
T he electrocardiogram is among the most common clinical tests ordered to assess cardiac abnormalities. Reproducible waveforms indicating discrete electrophysiologic processes were described over 100 years ago, yet the biological underpinnings of conduction and repolarization remain incompletely defined. The electrocardiographic PR interval reflects conduction from the atria to ventricles, across specialized conduction tissues such as the atrioventricular node and the His-Purkinje system. Pathological variation in the PR interval may indicate heart block or pre-excitation, both of which can lead to sudden death ${ }^{1}$. The PR interval also serves as a risk factor for atrial fibrillation and cardiovascular mortality ${ }^{1-3}$. Prior genetic association studies have identified $64 \mathrm{PR}$ interval loci ${ }^{4-13}$. Yet the underlying biological mechanisms of atrioventricular conduction and relationships between genetic predisposition to PR interval duration and disease are incompletely characterized.

To enhance our understanding of the genetic and biological mechanisms of atrioventricular conduction, we perform genomewide association studies (GWAS) meta-analyses of autosomal and $\mathrm{X}$ chromosome variants mainly imputed with the 1000 Genomes Project reference panel (http://www.internationalgenome.org) $)^{14}$ of PR interval duration. We then conduct downstream in silico analyses to elucidate candidate genes and key pathways, and examine relationships between genetic variants linked to PR interval duration and cardiovascular disease in the UK biobank (UKB; https://www.ukbiobank.ac.uk). Over 200 loci are genomewide significant, and our results imply key regulation processes for atrioventricular conduction, and candidate genes in cardiac muscle development/contraction and the cytoskeleton. We observe associations between polygenic predisposition to PR interval duration with distal conduction disease, $\mathrm{AF}$, and atrioventricular pre-excitation. Our findings highlight the polygenic basis of atrioventricular conduction, and the genetic relationship between PR interval duration and other cardiovascular diseases.

\section{Results}

Meta-analysis of GWASs. We performed a primary metaanalysis including 293,051 individuals of European (92.6\%), African (2.7\%), Hispanic (4\%), and Brazilian (<1\%) ancestries from 40 studies (Supplementary Data 1 and 2, Supplementary Table 1). We also performed ancestry-specific meta-analyses (Fig. 1). A total of 202 genome-wide significant loci $\left(P<5 \times 10^{-8}\right)$ were identified in the multi-ancestry analysis, of which 141 were not previously reported (Supplementary Data 3, Fig. 2, Supplementary Figs. 1 and 2). We considered for discovery only variants present in $>60 \%$ of the maximum sample size in the GWAS summary results, a filtering criterion used to ensure robustness of associated loci (median proportion of sample size included in analyses for lead variants 1.0, interquartile range 0.99-1.00; Methods). There was strong support in our data for all 64 previously reported loci $\left(61\right.$ at $P<5 \times 10^{-8}$ and 3 at $P<1.1 \times 10^{-4}$; Supplementary Data 4 and 5). In a secondary analysis among the European ancestry subset, a total of 127 loci not previously reported reached genome-wide significance (Supplementary Data 6, Supplementary Figs. 1-4), of which lead variants at 8 loci were borderline genome-wide significant $\left(P<9.1 \times 10^{-7}\right)$ in our multi-ancestry meta-analysis. None of the previously unreported loci were genome-wide significant in African or Hispanic/Latino ancestry meta-analyses (Supplementary Data 7, Supplementary Figs. 1 and 3). We observed no genome-wide significant loci in the $\mathrm{X}$ chromosome meta-analyses (Supplementary Fig. 5). In sensitivity analyses, we examined the rank-based inverse normal transformed residuals of $\mathrm{PR}$ interval. Results of absolute and transformed trait meta-analyses were highly correlated $(P>0.94$, Supplementary Data 8-10, Supplementary Figs. 6 and 7).
By applying joint and conditional analyses in the European meta-analysis data, we identified multiple independently associated variants $\left(P_{\text {joint }}<5 \times 10^{-8}\right.$ and $\left.r^{2}<0.1\right)$ at 12 previously not reported and 25 previously reported loci (Supplementary Data 11). The overall variant-based heritability $\left(h_{\mathrm{g}}^{2}\right)$ for the PR interval estimated in 59,097 unrelated European participants from the UKB with electrocardiograms was $18.2 \%$ (Methods). In the UKB, the proportion of $h^{2}$ explained by variation at all loci discovered in our analysis was $62.6 \%$, compared with $33.5 \%$ when considering previously reported loci only.

We annotated variants at 149 loci (141 previously not reported loci from the multi-ancestry meta-analysis and 8 loci from the meta-analysis of European ancestry subset). The majority of the lead variants at the 149 loci were common (minor allele frequency, $\mathrm{MAF}>5 \%$ ). We observed 6 low-frequency (MAF $1-5 \%)$ variants, and one rare $(\mathrm{MAF}<1 \%)$ predicted damaging missense variant. The rare variant (rs35816944, p.Ser171Leu) is in SPSB3 encoding SplA/Ryanodine Receptor Domain and SOCS Box-containing 3. SPSB3 is involved in degradation of the transcription factor SNAIL, which regulates the epithelialmesenchymal transition ${ }^{15}$, and has not been previously associated with cardiovascular traits. At MYH6, a previously described locus for PR interval ${ }^{6,10}$, sick sinus syndrome ${ }^{16}, \mathrm{AF}$ and other cardiovascular traits ${ }^{17}$, we observed a previously not reported predicted damaging missense variant in MYH6 (rs28711516, p. Gly56Arg). MYH6 encodes the a-heavy chain subunit of cardiac myosin. In total, we identified missense variants in genes at 11 previously not reported loci, one from the European subset meta-analysis, and 6 previously reported loci (Supplementary Data 12). These variants are a representation of multiple variants at each locus, which are in high LD, and thus may not be the causative variant.

Expression quantitative trait loci (eQTLs). PR interval lead variants (or best proxy $\left[r^{2}>0.8\right]$ ) at 43 previously not reported and 23 previously reported loci were significant cis-eQTLs (at a $5 \%$ false discovery rate (FDR) in left ventricle (LV) and right atrial appendage (RAA) tissue samples from the Genotype-Tissue Expression (GTEx; https://gtexportal.org/home/) project ${ }^{18}$. Variants at 13 previously not reported and 6 previously reported loci were eQTLs in spleen, which was used as negative control tissue (Supplementary Data 13). The PR interval associations and eQTLs colocalized at 31 previously not reported loci and 14 previously reported loci (posterior probability $[\mathrm{PP}]>75 \%$. Variants at 9 previously not reported loci were significant eQTLs only in LV and RAA tissues with consistent directionality of gene expression.

Predicted gene expression. In an exploratory analysis, we also performed a transcriptome-wide analysis to evaluate associations between predicted gene expression in LV and RAA with the PR interval. We identified 113 genes meeting our significance threshold $\left(P<3.1 \times 10^{-6}\right.$, after Bonferroni correction), of which 91 were localized at PR interval loci (within $500 \mathrm{~kb}$ from a lead variant; Supplementary Data 14, Supplementary Fig. 8). Longer PR interval duration was associated with decreased levels of predicted gene expression for 57 genes, and increased levels for 56 genes (Fig. 3). In spleen tissues, only 31 gene expression-PR interval associations were detected, and 19 of them did not overlap with the findings in heart tissues.

Regulatory annotation of loci. Most $\mathrm{PR}$ interval variants were annotated as non-coding. Therefore, we explored whether associated variants or proxies were located in transcriptionally active genomic regions. We observed enrichment for DNase 


\begin{tabular}{|c|c|c|c|c|}
\hline $\begin{array}{l}\text { Contributing } \\
\text { studies }\end{array}$ & \multicolumn{3}{|c|}{$\begin{array}{c}\text { CHARGE consortium member studies }(N=152,423) \\
\text { deCODE }(N=80,085) \\
\text { UK Biobank }(N=60,543)\end{array}$} & \\
\hline \multirow[t]{2}{*}{$\begin{array}{l}\text { Single stage } \\
\text { discovery }\end{array}$} & $\begin{array}{r}\text { Autosomes } \\
\text { Multi-ancestry }(N=29 \\
\text { European ancestry }(N= \\
\text { African ancestry }(N= \\
\text { Hispanic/Latino ancestry }(\end{array}$ & $\begin{array}{l}1)^{\star} \\
570) \\
3) \\
2,823)\end{array}$ & \multicolumn{2}{|c|}{$\begin{array}{l}\text { Chromosome } X \\
\text { Multi-ancestry }\left(N_{\text {males }}=101,170 ; N_{\text {females }}=112,093\right)^{*} \\
\text { European ancestry }\left(N_{\text {males }}=99,706 ; N_{\text {females }}=109,745\right) \\
\text { African ancestry }\left(N_{\text {males }}=1,245 ; N_{\text {females }}=2,082\right)\end{array}$} \\
\hline & \multicolumn{4}{|c|}{$\begin{array}{l}202 \text { PR interval loci at } P<5 \times 10^{-8} \text { ( } 141 \text { previously not reported) } \\
\text { from multi-ancestry meta-analysis }\end{array}$} \\
\hline \multirow[t]{2}{*}{$\begin{array}{l}\text { Bioinformatics \& } \\
\text { in silico functional } \\
\text { annotations }^{\#}\end{array}$} & $\begin{array}{l}\text { Functional annotation } \\
\text { of variants }\end{array}$ & $\begin{array}{l}\text { Effect of variants on } \\
\text { gene expression }\end{array}$ & $\begin{array}{l}\text { Target genes of } \\
\text { regulatory variants }\end{array}$ & $\begin{array}{l}\text { Geneset enrichment } \\
\text { \& pathway analyses }\end{array}$ \\
\hline & $\begin{array}{l}\text { - Coding variants (Variant } \\
\text { effect predictor) } \\
\text { - Noncoding variants } \\
\text { (HaploReg) }\end{array}$ & $\begin{array}{l}\text { - eQTLs } \\
\text { - Predicted gene } \\
\text { expression (S- } \\
\text { PrediXcan) }\end{array}$ & $\begin{array}{l}\text { - Long-range } \\
\text { chromatin } \\
\text { interaction }(\mathrm{Hi}-\mathrm{C})\end{array}$ & $\begin{array}{l}\text { - DEPICT } \\
\text { - Ingenuity Pathway } \\
\text { Analysis }\end{array}$ \\
\hline $\begin{array}{l}\text { Disease/trait } \\
\text { associations }\end{array}$ & $\begin{array}{l}\text { - Polygenic risk } \\
\text { - Look-ups at pi }\end{array}$ & $\begin{array}{l}\text { analyses with cardiova } \\
\text { GWAS databases (Phen }\end{array}$ & $\begin{array}{l}\text { cular outcomes in UK Bi } \\
\text { Scanner \& GWAS catalc }\end{array}$ & \\
\hline
\end{tabular}

Fig. 1 Overview of the study design. An overview of contributing studies, single-stage discovery approach, and downstream bioinformatics and in silico annotations performed to link variants to genes, and polygenic risk score analysis to link variants to cardiovascular disease risk is illustrated. Asterisk ( $\left.{ }^{\star}\right)$ The multi-ancestry meta-analysis is our primary analysis. Previously not reported loci were identified from the multi-ancestry meta-analysis. Ancestry specific and chromosome X meta-analysis are secondary. Hash $\left.{ }^{\#}\right)$ For bioinformatics and in silico annotations we also included loci that reached genome-wide significance in European only meta-analysis $(\mathrm{N}=8)$ and were borderline genome-wide significant in the multi-ancestry meta-analysis.

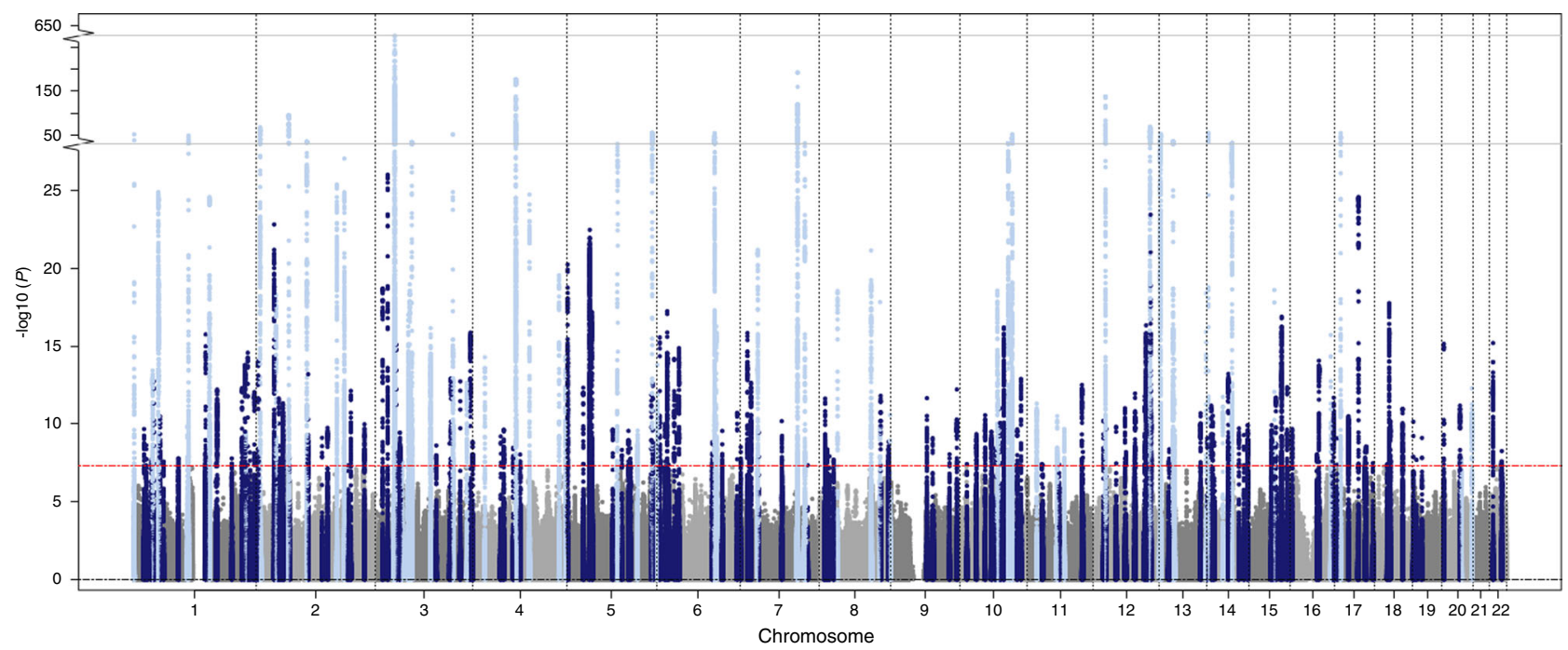

Fig. 2 Manhattan plot of the multi-ancestry meta-analysis for PR interval. $P$ values are plotted on the $-\log _{10}$ scale for all variants present in at least $60 \%$ of the maximum sample size from the fixed-effects meta-analysis of 293,051 individuals from multiple ancestries (multi-ancestry meta-analysis). Associations of genome-wide significant $\left(P<5 \times 10^{-8}\right)$ variants at previously not reported $(N=141)$ and previously reported loci $(N=61)$ are plotted in dark and light blue colors respectively.

I-hypersensitive sites in fetal heart tissue $\left(P<9.36 \times 10^{-5}\right.$, Supplementary Fig. 9). Analysis of chromatin states indicated variants at 97 previously not reported, 6 European, and 52 previously reported loci were located within regulatory elements that are present in heart tissues (Supplementary Data 15), providing support for gene regulatory mechanisms in specifying the PR interval. To identify distal candidate genes at PR interval loci, we assessed the same set of variants for chromatin interactions in a LV tissue Hi-C dataset ${ }^{19}$. Forty-eight target genes were identified (Supplementary Data 16). Variants at 35 previously not reported and 3 European loci were associated with other traits, including AF and coronary heart disease (Supplementary Data 17, Supplementary Fig. 10). 

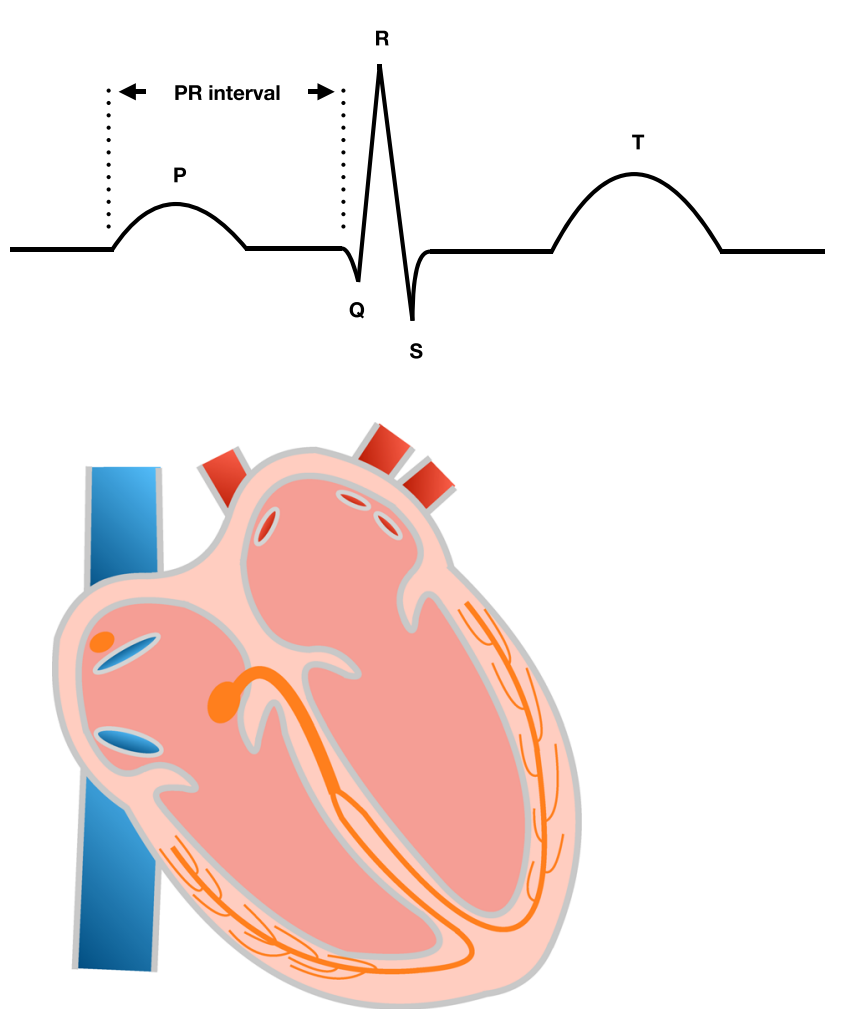

Gene expression related to longer PR

$\begin{array}{cccc}\text { ACP6 } & \text { DNM1P51 } & \text { MYO15A } & \text { TCTN3 } \\ \text { AL590822.1 } & \text { EDN2 } & \text { NPIPA5 } & \text { TMEM182 } \\ \text { ALPK3 } & \text { EFNA1 } & \text { NUDT13 } & \text { TPMT } \\ \text { ATP5D } & \text { FADS1 } & \text { PDZRN3 } & \text { TRAK1 } \\ \text { BMPR1A } & \text { FAM211B } & \text { PHACTR1 } & \text { TRIP4 } \\ \text { C11orf1 } & \text { FAT1 } & \text { RP11-29H23.5 } & \text { TTC18 } \\ \text { CALHM2 } & \text { FKBP7 } & \text { RP11-399K21.11 } & \text { VDAC2 } \\ \text { CAMK2D } & \text { FUT11 } & \text { RP11-3B7.1 } & \text { VPREB3 } \\ \text { CCDC36 } & \text { GBAP1 } & \text { RP4-764O22.2 } & \text { XIRP1 } \\ \text { CDH13 } & \text { HMGA1P5 } & \text { RPSA } & \text { ZCCHC24 } \\ \text { CEFIP } & \text { IFRD2 } & \text { SLC25A26 } & \text { ZNF503-AS1 } \\ \text { CFDP1 } & \text { KCND3 } & \text { SLC6A6 } & \\ \text { CHRM2 } & \text { KDM1B } & \text { SLK } & \\ \text { DAG1 } & \text { LRCH1 } & \text { SNX1 } & \\ \text { DEK } & \text { MSTO2P } & \text { SYNPO2L } & \end{array}$

Gene expression related to shorter PR

$\begin{array}{cccc}\text { AC011747.4 } & \text { IL17D } & \text { PLCD1 } & \text { SPATA20 } \\ \text { AC103965.1 } & \text { IL25 } & \text { PPAPDC3 } & \text { SPTBN1 } \\ \text { AGAP5 } & \text { KPNA3 } & \text { QRICH1 } & \text { SSBP3 } \\ \text { BEND7 } & \text { LINC00964 } & \text { RCAN2 } & \text { SSXP10 } \\ \text { C10rf86 } & \text { MALAT1 } & \text { RP11-1070N10.3 } & \text { STRN } \\ \text { CAB39L } & \text { MLF1 } & \text { RP11-182J1.16 } & \text { SYNE2 } \\ \text { CBX8 } & \text { MMP11 } & \text { RP11-344N10.5 } & \text { SYPL2 } \\ \text { CMTM5 } & \text { MRPL37 } & \text { RP11-379F4.7 } & \text { TFEC } \\ \text { CSPG4P11 } & \text { MTSS1 } & \text { RP11-397E7.4 } & \text { THRB } \\ \text { DDX42 } & \text { MYBPHL } & \text { RP11-724N1.1 } & \text { UBE3B } \\ \text { DNAH11 } & \text { MYOZ1 } & \text { SCN5A } & \text { WDR73 } \\ \text { EMB } & \text { NDST2 } & \text { SCN10A } & \text { ZHX1 } \\ \text { GBF1 } & \text { NEURL } & \text { SH3PXD2A } & \\ \text { GORASP1 } & \text { NPIPA1 } & \text { SLC2A11 } & \\ \text { HCN1 } & \text { PHLDB2 } & \text { SMARCB1 } & \end{array}$

Fig. 3 Plausible candidate genes of PR interval from S-PrediXcan. Diagram of standard electrocardiographic intervals and the heart. The electrocardiographic features are illustratively aligned with the corresponding cardiac conduction system structures (orange) reflected on the tracing. The PR interval (labeled) indicates conduction through the atria, atrioventricular node, His bundle, and Purkinje fibers. Right: Supplementary Data 14 shows 113 genes whose expression in the left ventricle $(N=233)$ or right atrial appendage $(N=231)$ was associated with PR interval duration in a transcriptome-wide analysis using S-PrediXcan and GTEx v7. Displayed genes include those with significant associations after Bonferroni correction for all tested genes $(P<$ $3.1 \times 10^{-6}$ ). Longer PR intervals were associated with increased predicted expression of 56 genes (blue) and reduced expression of 57 genes (orange).

In silico functional annotation and pathway analysis. Bioinformatics and in silico functional annotations for potential candidate genes at the 149 loci are summarized in Supplementary Data 18 and 19. Using a prior GWAS of $\mathrm{AF}^{20,21}$, we identified variants with shared associations between PR interval duration and AF risk (Supplementary Fig. 11). Enrichment analysis of genes at PR interval loci using Data driven Expression-Prioritized Integration for Complex Traits (DEPICT: https://data. broadinstitute.org $/ \mathrm{mpg} / \mathrm{depict} /)^{22}$ indicated heart development $\left(P=1.87 \times 10^{-15}\right)$ and actin cytoskeleton organization $(P=$ $2.20 \times 10^{-15}$ ) as the most significantly enriched processes (Supplementary Data 20 and 21). Ingenuity Pathway Analysis (IPA; https://www.qiagenbioinformatics.com/products/ingenuity-

pathway-analysis/) supported heart development, ion channel signaling and cell-junction/cell-signaling amongst the most significant canonical pathways (Supplementary Data 22).

Polygenic risk scores (PRSs) with cardiovascular traits. Finally, we evaluated associations between genetic predisposition to PR interval duration and 16 cardiac phenotypes chosen a priori using $\sim 309,000$ unrelated UKB European participants not included in our meta-analyses ${ }^{23}$. We created a PRS for PR interval using the European ancestry meta-analysis results (Fig. 4, Supplementary Table 2). Genetically determined PR interval prolongation was associated with higher risk of distal conduction disease (atrioventricular block; odds ratio [OR] per standard deviation 1.11, $P=7.02 \times 10^{-8}$ ) and pacemaker implantation (OR 1.06, $P=$ $\left.1.5 \times 10^{-4}\right)$. In contrast, genetically determined PR interval prolongation was associated with reduced risk of $\mathrm{AF}$ (OR $0.95, \mathrm{P}=$ $4.30 \times 10^{-8}$ ) and was marginally associated with a reduced risk of atrioventricular pre-excitation (Wolff-Parkinson-White syndrome; OR $0.85, P=0.003)$. Results were similar when using a PRS derived using the multi-ancestry meta-analysis results (Supplementary Fig. 12, Supplementary Table 2, and Supplementary Data 3).

\section{Discussion}

In a meta-analysis of nearly 300,000 individuals, we identified 202 loci, of which 141 were previously not reported underlying cardiac conduction as manifested by the electrocardiographic PR interval. Apart from confirming well-established associations in loci harboring ion-channel genes, our findings further underscore the central importance of heart development and cytoskeletal components in atrioventricular conduction ${ }^{10,12,13}$. We also highlight the role of common variation at loci harboring genes underlying monogenic forms of arrhythmias and cardiomyopathies in cardiac conduction.

We report signals in/near 12 candidate genes at previously not reported loci with functional roles in cytoskeletal assembly $(D S P$, DES, OBSL1, PDLIM5, LDB3, FHL2, CEFIP, SSPN, TLN2, PTK2, GJA5, and CDH2; Fig. 5). DSP and DES encode components of the cardiac desmosome, a complex involved in ionic communication between cardiomyocytes and maintenance of cellular integrity. Mutations in the desmosome are implicated in arrhythmogenic cardiomyopathy (ACM) and dilated cardiomyopathy (DCM $)^{24-28}$. Conduction slowing is a major component of the pathophysiology of arrhythmia in ACM and other cardiomyopathies ${ }^{29,30}$. OBSL1 encodes obscurin-like 1, which together with obscurin (OBSCN) is involved in sarcomerogenesis by bridging titin (TTN) and myomesin at the M-band ${ }^{31}$. PDLIM5 


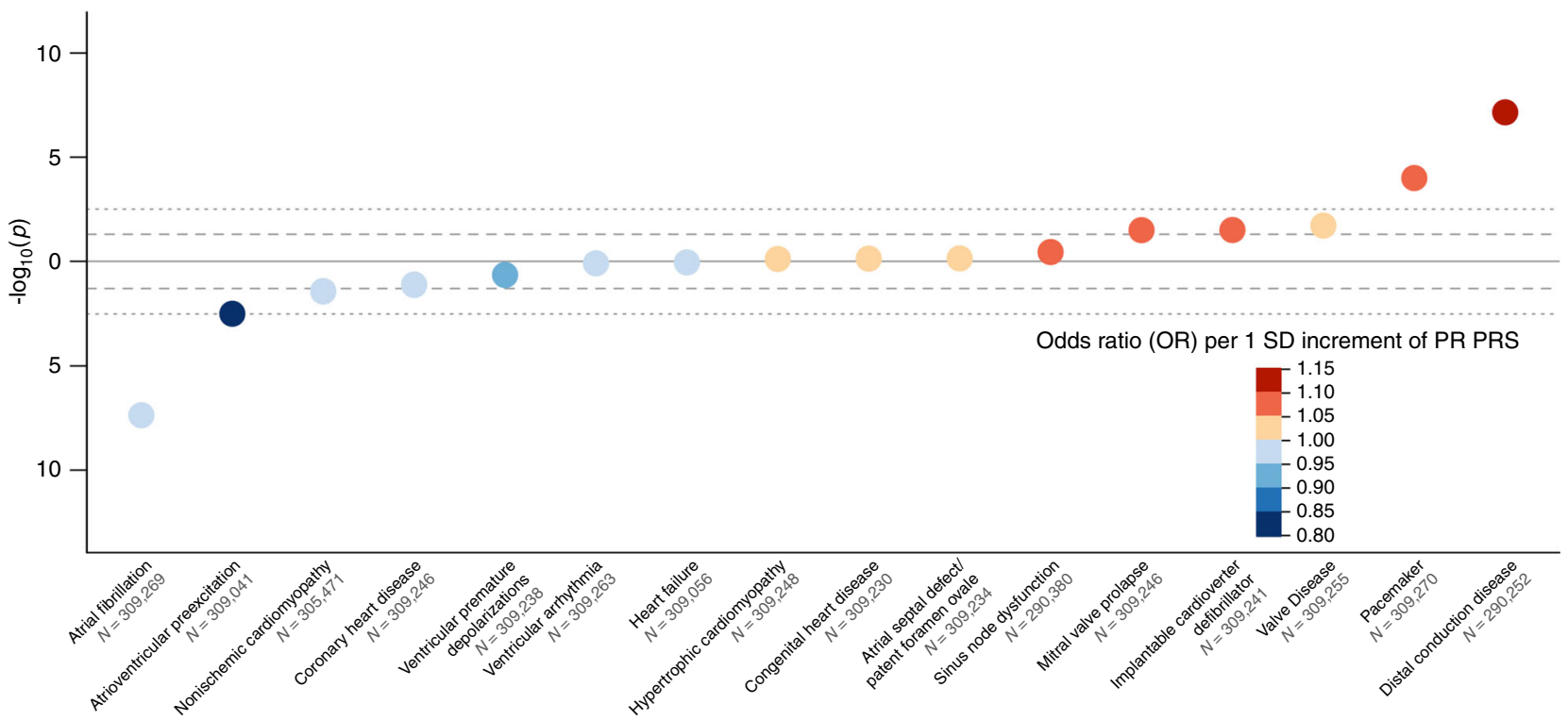

Fig. 4 Bubble plot of phenome-wide association analysis of European ancestry PR interval polygenic risk score. The polygenic risk score was derived from the European ancestry meta-analysis. Orange circles indicate that polygenic predisposition to longer PR interval is associated with an increased risk of the condition, whereas blue circles indicate that polygenic predisposition to longer PR interval is associated with lower risk of the condition. The darkness of the color reflects the effect size (odds ratio, OR) per 1 standard deviation (s.d.) increment of the polygenic risk score from logistic regression. Sample size $(N)$ in each regression model is provided under $X$-axis. Given correlation between traits, we set significance threshold at $P<3.13 \times 10^{-3}$ after Bonferroni correction $(P<0.05 / 16$; dotted line) for the analysis and also report nominal associations $(P<0.05$; dashed line $)$.

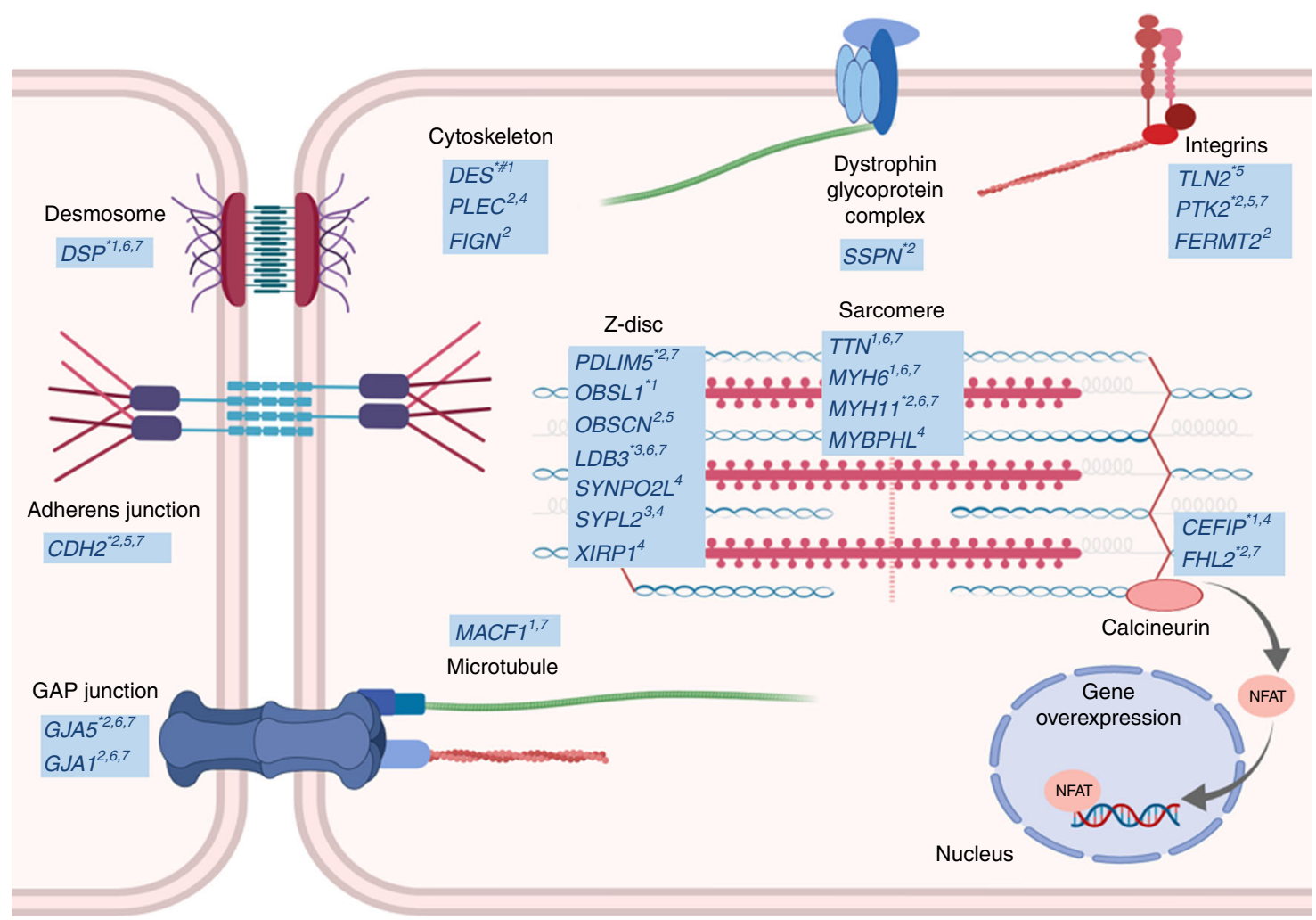

Fig. 5 Candidate genes in PR interval loci encoding proteins involved in cardiac muscle cytoskeleton. Candidate genes or encoded proteins are indicated by a star symbol in the figure and are listed in Supplementary Data 3. More information about the genes is provided in Supplementary Data 18 and 19 . This figure was created with BioRender. *Previously not reported locus, \# genome-wide significant locus in transformed trait meta-analysis. ${ }^{1} \mathrm{Missense}$ variant; ${ }^{2}$ Nearest gene to the lead variant; ${ }^{3}$ Gene within the region $\left(r^{2}>0.5\right) ;{ }^{4}$ Variant(s) in the locus are associated with gene expression in left ventricle and/or right atrial appendage; ${ }^{5}$ Left ventricle best HiC locus interactor (RegulomeDB score $\leq 2$ ); ${ }^{6}$ Animal model; ${ }^{7}$ Monogenic disease with a cardiovascular phenotype. 
encodes a scaffold protein that tethers protein kinases to the Zdisk, and has been associated with DCM in homozygous murine cardiac knockouts ${ }^{32}$. FHL2 encodes calcineurin-binding protein four and a half LIM domains 2, which is involved in cardiac development by negatively regulating calcineurin/NFAT signaling in cardiomyocytes ${ }^{33}$. Missense mutations in FHL2 have been associated with hypertrophic cardiomyopathy ${ }^{34}$. CEFIP encodes the cardiac-enriched FHL2-interacting protein located at the Zdisc, which interacts with FHL2. It is also involved in calcineurin-NFAT signaling, but its overexpression leads to cardiomyocyte hypertrophy ${ }^{35}$.

Common variants in/near genes associated with monogenic arrhythmia syndromes were also observed, suggesting these genes may also affect atrioventricular conduction and cardiovascular pathology in the general population. Apart from DSP, DES, and GJA5 discussed above, our analyses indicate 2 additional candidate genes (HCN4 and RYR2). HCN4 encodes a component of the hyperpolarization-activated cyclic nucleotide-gated potassium channel which specifies the sinoatrial pacemaker "funny" current, and is implicated in sinus node dysfunction, AF, and left ventricular noncompaction ${ }^{36-38}$. RYR2 encodes a calcium channel component in the cardiac sarcoplasmic reticulum and is implicated in catecholaminergic polymorphic ventricular tachycardia ${ }^{39}$.

Genes with roles in autonomic signaling in the heart (CHRM2, $A D C Y 5$ ) were indicated from expression analyses (Supplementary Data 13 and 18). CHRM2 encodes the M2 muscarinic cholinergic receptors that bind acetylcholine and are expressed in the heart ${ }^{40}$. Their stimulation results in inhibition of adenylate cyclase encoded by $A D C Y 5$, which in turn inhibits ion channel function. Ultimately, the signaling cascade can result in reduced levels of the pacemaker "funny" current in the sinoatrial and atrioventricular nodes, reduced L-type calcium current in all myocyte populations, and increased inwardly rectifying $I_{\text {K.Ach }}$ potassium current in the conduction tissues and atria causing cardiomyocyte hyperpolarization ${ }^{41}$. Stimulation has also been reported to shorten atrial action potential duration and thereby facilitate reentry, which may lead to $\mathrm{AF}^{42-44}$.

By constructing PRSs, we also observed that genetically determined PR interval duration is an endophenotype for several adult-onset complex cardiovascular diseases, the most significant of which are arrhythmias and conduction disorders. For example, our findings are consistent with previous epidemiologic data supporting a U-shaped relationship between PR interval duration and $\mathrm{AF}$ risk $^{2}$. Although aggregate genetic predisposition to $\mathrm{PR}$ interval prolongation is associated with reduced AF risk, top PR interval prolonging alleles are associated with decreased $\mathrm{AF}$ risk (e.g., localized to the $S C N 5 A / S C N 10 A$ locus; Supplementary Fig. 11) whereas others are associated with increased AF risk (e.g., localized to the TTN locus; Supplementary Fig. 11), consistent with prior reports ${ }^{8}$. These findings suggest that genetic determinants of the PR interval may identify distinct pathophysiologic mechanisms leading to AF, perhaps via specifying differences in tissue excitability, conduction velocity, or refractoriness. Future efforts are warranted to better understand the relations between genetically determined PR interval and specific arrhythmia mechanisms.

In conclusion, our study more than triples the reported number of PR interval loci, which collectively explain $\sim 62 \%$ of trait-related heritability. Our findings highlight important biological processes underlying atrioventricular conduction, which include both ion channel function, and specification of cytoskeletal components. Our study also indicates that common variation in Mendelian cardiovascular disease genes contributes to population-based variation in the PR interval. Lastly, we observe that genetic determinants of the PR interval provide novel insights into the etiology of several complex cardiac diseases, including AF. Collectively, our results represent a major advance in understanding the polygenic nature of cardiac conduction, and the genetic relationship between PR interval duration and arrhythmias.

\section{Methods}

Contributing studies. A total of 40 studies (Supplementary Methods) comprising 293,051 individuals of European $(N=271,570)$, African $(N=8,173)$, Hispanic $(N=11,686)$, and Brazilian $(N=485)$ ancestries contributed GWAS summary statistics for PR interval. Study-specific design, sample quality control and descriptive statistics are provided in Supplementary Tables 1-3. For the majority of the studies imputation was performed for autosomal chromosomes and X chromosome using the 1000 Genomes (1000 G: http://www.internationalgenome.org) project $^{14}$ reference panel. A few studies used whole genome sequence data and the Haplotype Reference Consortium (HRC: http://www.haplotype-referenceconsortium.org)/UK10K and $1000 \mathrm{G}$ phase 3 panel was used for UK Biobank (Full details are provided in Supplementary Table 2).

Ethical approval. All contributing studies had study-specific ethical approvals and written informed consent. The details are provided in Supplementary Note 1.

PR interval phenotype and exclusions. The PR interval was measured in milliseconds (ms) from standard 12-lead electrocardiograms (ECGs), except in the UK Biobank where it was obtained from 4-lead ECGs (CAM-USB 6.5, Cardiosoft v6.51) recorded during a 15 second rest period prior to an exercise test (Supplementary Methods). We requested exclusion of individuals with extreme PR interval values ( $<80 \mathrm{~ms}$ or $>320 \mathrm{~ms}$ ), second/third degree heart block, AF on the ECG, or a history of myocardial infarction or heart failure, Wolff-Parkinson-White syndrome, those who had a pacemaker, individuals receiving class I and class III antiarrhythmic medications, digoxin, and pregnancy. Where data were available these exclusions were applied.

Study-level association analyses. We regressed the absolute PR interval on each genotype dosage using multiple linear regression with an additive genetic effect and adjusted for age, sex, height, body mass index, heart rate and any other studyspecific covariates. To account for relatedness, linear mixed effects models were used for family studies. To account for population structure, analyses were also adjusted for principal components of ancestry derived from genotyped variants after excluding related individuals. Analyses of autosomal variants were conducted separately for each ancestry group. X chromosome analyses were performed separately for males and females. Analyses using rank-based inverse normal transformed residuals of PR interval corrected for the aforementioned covariates were also conducted. Residuals were calculated separately by ancestral group for autosomal variants, and separately for males and females for X chromosome variants.

Centralized quality control. We performed quality control centrally for each result file using EasyQC version 11.4 (https://www.uni-regensburg.de/medizin/ epidemiologie-praeventivmedizin/genetische-epidemiologie/software/\#) ${ }^{45}$. We removed variants that were monomorphic, had a minor allele count $(\mathrm{MAC})<6$, imputation quality metric $<0.3$ (imputed by $\mathrm{MACH}$; http://csg.sph.umich.edu/ abecasis/mach/tour/imputation.html) or 0.4 (imputed by IMPUTE2; http:// mathgen.stats.ox.ac.uk/impute/impute_v2.html), had invalid or mismatched alleles, were duplicated, or if they were allele frequency outliers (difference $>0.2$ from the allele frequency in $1000 \mathrm{G}$ project). We inspected PZ plots, effect allele frequency plots, effect size distributions, QQ plots, and compared effect sizes in each study to effect sizes from prior reports for established PR interval loci to identify genotype and study-level anomalies. Variants with effective MAC $(=2 \times \mathrm{N} \times \mathrm{MAF} \times$ imputation quality metric) $<10$ were omitted from each study prior to metaanalysis.

Meta-analyses. We aggregated summary-level associations between genotypes and absolute PR interval from all individuals $(N=293,051)$, and only from Europeans $(N=271,570)$, African Americans $(N=8,173)$, and Hispanic/Latinos $(N=$ $12,823)$ using a fixed-effects meta-analysis approach implemented in METAL (http://csg.sph.umich.edu/abecasis/metal/, release on 2011/03/25) ${ }^{46}$. We considered as primary our multi-ancestry meta-analysis, and ancestry-specific metaanalyses as secondary. For the $\mathrm{X}$ chromosome, meta-analyses were conducted in a sex-stratified fashion. Genomic control was applied (if inflation factor $\lambda_{\mathrm{GC}}>1$ ) at the study level. Quantile-quantile (QQ) plots of observed versus expected $-\log _{10}(\mathrm{P})$ did not show substantive inflation (Supplementary Figs. 1 and 2).

Given the large sample size we undertook a one-stage discovery study design. To ensure the robustness of this approach we considered for discovery only variants reaching genome-wide significance $\left(P<5 \times 10^{-8}\right)$ present in at least $60 \%$ of the maximum sample size $\left(N_{\max }\right)$ in our GWAS summary results. We denote loci as previously not reported if the variants map outside 64 previously reported loci (Supplementary Methods, Supplementary Data 4) for both the multi-ancestry 
and ancestry-specific meta-analysis (secondary meta-analyses). Genome-wide significant variants were grouped into independent loci based on both distance $( \pm 500 \mathrm{~kb})$ and linkage disequilibrium (LD, $\left.r^{2}<0.1\right)$ (Supplementary Methods). We assessed heterogeneity in allelic effect sizes among studies contributing to the metaanalysis and among ancestral groups by the $\mathrm{I}^{2}$ inconsistency index ${ }^{47}$ for the lead variant in each previously not reported locus. LocusZoom (http://locuszoom.org/) ${ }^{48}$ was used to create region plots of identified loci. For reporting, we only declare as previously not reported genome-wide significant loci from our primary metaanalysis. However, we considered ancestry-specific loci for annotation and downstream analyses. The results from secondary analyses are specifically indicated in Supplementary Data 6 and 7.

Meta-analyses (multi-ancestry [ $N=282,128]$, European only $[N=271,570]$, and African $[\mathrm{N}=8,173]$ ) of rank-based inverse normal transformed residuals of PR interval were also performed (sensitivity meta-analyses). Because not all studies contributed summary-level association statistics of the transformed PR interval, we considered as primary the multi-ancestry meta-analysis of absolute PR interval for which we achieved the maximum sample size. Loci that met our significance criteria in the meta-analyses of transformed PR interval were not taken forward for downstream analyses.

Conditional and heritability analysis. Conditional and joint GWAS analyses were implemented in GCTA v1.91.3 (https://cnsgenomics.com/software/gcta/ \#Overview ${ }^{49}$ using summary-level variant statistics from the European ancestry meta-analysis to identify independent association signals within PR interval loci. We used 59,097 unrelated (kinship coefficient $>0.0884$ ) UK Biobank participants of European ancestry as the reference sample to model patterns of LD between variants. We declared as conditionally independent any genome-wide significant variants in conditional analysis $\left(P_{\text {joint }}<5 \times 10^{-8}\right)$ not in $\operatorname{LD}\left(r^{2}<0.1\right)$ with the lead variant in the locus.

Using the same set of individuals from UK Biobank, we estimated the aggregate genetic contributions to PR interval with restricted maximum likelihood as implemented in BOLT-REML v2.3.4 (https://data.broadinstitute.org/alkesgroup/ BOLT-LMM $/)^{50}$. We calculated the additive overall variant-heritability $\left(h^{2}\right)$ based on 333,167 LD-pruned genotyped variants, as well as the $h^{2}$ g of variants at PR interval associated loci only. Loci windows were based on both distance $( \pm 500 \mathrm{~kb})$ and $\mathrm{LD}\left(r^{2}>0.1\right)$ around previously not reported and reported variants (Supplementary Methods). We then calculated the proportion of total $h^{2} \mathrm{~g}$ explained at PR interval loci by dividing the $h^{2}$ estimate of PR interval loci by the total $h^{2}$.

Bioinformatics and in silico functional analyses. We use Variant Effect Predictor (VEP; https://www.ensembl.org/info/docs/tools/vep/index.html) ${ }^{51}$ to obtain functional characterization of variants including consequence, information on nearest genes and, where applicable, amino acid substitution and functional impact, based on SIFT ${ }^{52}$ and PolyPhen- $2^{53}$ prediction tools. For non-coding variants, we assessed overlap with DNase I-hypersensitive sites (DHS) and chromatin states as determined by Roadmap Epigenomics Project ${ }^{54}$ across all tissues and in cardiac tissues (E083, fetal heart; E095, LV; E104, right atrium; E105, right ventricle) using HaploReg v4.1 (https://pubs.broadinstitute.org/mammals/haploreg/haploreg.php) ${ }^{55}$ and using FORGE (https://github.com/iandunham/Forge).

We assessed whether any PR interval variants were related to cardiac gene expression using GTEx (https://gtexportal.org/home/) ${ }^{18}$ version 7 cis-eQTL LV $(N=233)$ and RAA $(N=231)$ European data. If the variant at a locus was not available in GTEx, we used proxy variants $\left(r^{2}>0.8\right)$. We then evaluated the effects of predicted gene expression levels on PR interval duration using S-PrediXcan (https://github.com/hakyimlab/MetaXcan) ${ }^{56}$. GTEx ${ }^{18}$ genotypes (variants with MAF $>0.01$ ) and normalized expression data in LV and RAA provided by the software developers were used as the training datasets for the prediction models The prediction models between each gene-tissue pair were performed by ElasticNet, and only significant models for prediction were included in the analysis, where significance was determined if nested cross validated correlation between predicted and actual levels were greater than 0.10 (equivalent to $\mathrm{R}^{2}>0.01$ ) and $P$ value of the correlation test was less than 0.05 . We used the European meta-analysis summarylevel results (variants with at least $60 \%$ of maximum sample size) as the study dataset and then performed the S-PrediXcan calculator to estimate the expressionPR interval associations. For both eQTL and S-PrediXcan assessments, we additionally included spleen tissue in Europeans $(N=119)$ as a negative control. In total, we tested 5366, 5977, and 4598 associations in LV, RAA, and spleen, respectively. Significance threshold of S-PrediXcan was set at $P=3.1 \times 10^{-6}$ $(=0.05 /(5977+5366+4598))$ to account for multiple testing. In order to determine whether the GWAS identified loci were colocalized with the eQTL analysis, we performed genetic colocalization analysis for eQTL and S-PrediXcan identified gene regions, using the Bayesian approach in COLOC package ( $\mathrm{R}$ version 3.5; https://cran.r-project.org/web/packages/coloc/index.html). Variants located within the same identified gene regions were included. We set the significant threshold for the PP (two significant associations sharing a common causal variant) at $>75 \%$.

We applied GARFIELD (GWAS analysis of regulatory or functional information enrichment with LD correction; https://www.ebi.ac.uk/birney-srv/ GARFIELD/ $/)^{57}$ to analyze the enrichment patterns for functional annotations of the European meta-analysis summary statistics, using regulatory maps from the Encyclopedia of DNA Elements (ENCODE) ${ }^{58}$ and Roadmap Epigenomics ${ }^{54}$ projects. This method calculates odds ratios and enrichment $P$ values at different GWAS $P$ value thresholds (denoted T) for each annotation by using a logistic regression model accounting for $\mathrm{LD}$, matched genotyping variants and local gene density with the application of logistic regression to derive statistical significance. Threshold for significant enrichment was set to $P=9.36 \times 10^{-5}$ (after multipletesting correction for the number of effective annotations).

We identified potential target genes of regulatory variants using long-range chromatin interaction (Hi-C) data from the $\mathrm{LV}^{19}$. Hi-C data was corrected for genomic biases and distance using the Hi-C Pro and Fit-Hi-C pipelines according to Schmitt et al. ( $40 \mathrm{~kb}$ resolution - correction applied to interactions with $50 \mathrm{~kb}-5 \mathrm{Mb}$ span). We identified the promoter interactions for all potential regulatory variants in $\mathrm{LD}\left(r^{2}>0.8\right)$ with our lead and conditionally independent $\mathrm{PR}$ interval variants and report the interactors with the variants with the highest regulatory potential a Regulome DB score of $\leq 2$ (RegulomeDB; http://www. regulomedb.org) to annotate the loci.

We performed a literature review, and queried the Online Mendelian Inheritance in Man (OMIM; https://www.omim.org/) and the International Mouse Phenotyping Consortium (https://www.mousephenotype.org/) databases for all genes in regions defined by $r^{2}>0.5$ from the lead variant at each previously not reported locus. We further expanded the gene listing with any genes that were indicated by gene expression or chromatin interaction analyses. We performed look-ups for each lead variant or their proxies $\left(r^{2}>0.8\right)$ for associations $(P<5 \times$ $10^{-8}$ ) for common traits using both GWAS catalog ${ }^{59}$ and PhenoScanner v2 ${ }^{60}$ databases. For AF, we summarized the results of lead PR interval variants for PR interval and their associations with AF risk from two recently published $\mathrm{GWASs}^{20,21}$. We included variants in high linkage disequilibrium with lead PR variants $\left(r^{2}>0.7\right)$.

Geneset enrichment and pathway analyses. We used DEPICT (https://data. broadinstitute.org/mpg/depict/ $)^{22}$ to identify enriched pathways and tissues/cell types where genes from associated loci are highly expressed using all genome-wide significant $\left(P<5 \times 10^{-8}\right)$ variants in our multi-ancestry meta-analysis present in at least $60 \%$ of $N_{\max }\left(N_{\text {variants }}=20,076\right)$. To identify uncorrelated variants for PR interval, DEPICT performed LD-clumping $\left(r^{2}=0.1\right.$, window size $\left.=250 \mathrm{~kb}\right)$ using LD estimates between variants from the $1000 \mathrm{G}$ reference data on individuals from all ancestries after excluding the major histocompatibility complex region on chromosome 6. Geneset enrichment analysis was conducted based on 14,461 predefined reconstituted gene sets from various databases and data types, including Gene ontology, Kyoto encyclopedia of genes and genomes (KEGG), REACTOME, phenotypic gene sets derived from the Mouse genetics initiative, and protein molecular pathways derived from protein-protein interaction. Finally, tissue and cell type enrichment analyses were performed based on expression information in any of the 209 Medical Subject Heading (MeSH) annotations for the 37,427 human Affymetrix HGU133a2.0 platform microarray probes.

IPA (https://www.qiagenbioinformatics.com/products/ingenuity-pathwayanalysis/) was conducted using an extended list comprising 593 genes located in regions defined by $r^{2}>0.5$ with the lead or conditionally independent variants for all PR interval loci, or the nearest gene. We further expanded this list by adding genes indicated by gene expression analyses. Only molecules and/or relationships for human or mouse or rat and experimentally verified results were considered. The significance $P$ value associated with enrichment of functional processes is calculated using the right-tailed Fisher's exact test by considering the number of query molecules that participate in that function and the total number of molecules that are known to be associated with that function in the IPA.

Associations between genetically determined PR interval and cardiovascular conditions. We examined associations between genetic determinants of atrioven tricular conduction and candidate cardiovascular diseases in unrelated individuals of European ancestry from UK Biobank ( $N \sim 309,000$ not included in our GWAS meta-analyses) by creating PRSs for PR interval based on our GWAS results. We derived two PRSs. One was derived from the European ancestry meta-analysis results, and the other from the multi-ancestry meta-analysis results. We used the LD-clumping feature in PLINK v1.9061 $\left(r^{2}=0.1\right.$, window size $\left.=250 \mathrm{~kb}, P=5 \times 10^{-8}\right)$ to select variants for each PRS. Referent LD structure was based on $1000 \mathrm{G}$ European only, and all ancestry data. In total, we selected 582 and 743 variants from European only and multi-ancestry meta-analysis results, respectively. We calculated the PRSs for PR interval by summing the dosage of PR interval prolonging alleles weighted by the corresponding effect size from the meta-analysis results. A total of 581 variants for the PRS derived from European results and 743 variants for the PRS derived from multi-ancestry results (among the variants with imputation quality $>0.6)$ were included in our PRS calculations.

We selected candidate cardiovascular conditions a priori, which included various cardiac conduction and structural traits such as bradyarrhythmia, AF, atrioventricular pre-excitation, heart failure, cardiomyopathy, and congenital heart disease. We ascertained disease status based on data from baseline interviews, hospital diagnosis codes (ICD-9 and ICD-10), cause of death codes (ICD-10), and operation codes. Details of individual selections and disease definitions are described in Supplementary Data 23. 
We tested the PRSs for association with cardiovascular conditions using logistic regression. We adjusted for enrolled age, sex, genotyping array, and phenotyperelated principal components of ancestry. Given correlation between traits, we set significance threshold at $P<3.13 \times 10^{-3}$ after Bonferroni correction $(P<0.05 / 16)$ for the number of analyses performed and also report nominal associations $(P<0.05)$.

Reporting summary. Further information on research design is available in the Nature Research Reporting Summary linked to this article.

\section{Data availability}

Summary GWAS statistics are publicly available on the Cardiovascular Disease Knowledge portal (http://www.broadcvdi.org). All other data are contained in the article file and its supplementary information or is available upon request.

Received: 2 August 2019; Accepted: 18 March 2020;

Published online: 21 May 2020

\section{References}

1. Cheng, S. et al. Long-term outcomes in individuals with prolonged PR interval or first-degree atrioventricular block. JAMA 301, 2571-7 (2009).

2. Alonso, A. et al. Simple risk model predicts incidence of atrial fibrillation in a racially and geographically diverse population: the CHARGE-AF consortium. J. Am. Heart Assoc. 2, e000102 (2013).

3. Rasmussen, P. V. et al. Electrocardiographic PR Interval Duration and Cardiovascular Risk: Results From the Copenhagen ECG Study. Can. J. Cardiol. 33, 674-681 (2017).

4. Butler, A. M. et al. Novel loci associated with PR interval in a genome-wide association study of 10 African American cohorts. Circ. Cardiovasc. Genet. 5, 639-46 (2012).

5. Chambers, J. C. et al. Genetic variation in $\mathrm{SCN} 10 \mathrm{~A}$ influences cardiac conduction. Nat. Genet. 42, 149-52 (2010).

6. Holm, H. et al. Several common variants modulate heart rate, PR interval and QRS duration. Nat. Genet. 42, 117-22 (2010).

7. Hong, K. W. et al. Identification of three novel genetic variations associated with electrocardiographic traits (QRS duration and PR interval) in East Asians. Hum. Mol. Genet. 23, 6659-67 (2014).

8. Pfeufer, A. et al. Genome-wide association study of PR interval. Nat. Genet. 42, 153-9 (2010)

9. Sano, M. et al. Genome-wide association study of electrocardiographic parameters identifies a new association for PR interval and confirms previously reported associations. Hum. Mol. Genet. 23, 6668-76 (2014).

10. van Setten, J. et al. PR interval genome-wide association meta-analysis identifies 50 loci associated with atrial and atrioventricular electrical activity. Nat. Commun. 9, 2904 (2018).

11. Verweij, N. et al. Genetic determinants of $\mathrm{P}$ wave duration and PR segment. Circ. Cardiovasc. Genet. 7, 475-81 (2014)

12. van Setten, J. et al. Genome-wide association meta-analysis of 30,000 samples identifies seven novel loci for quantitative ECG traits. Eur. J. Hum. Genet. 27, 952-962 (2019).

13. Lin, $\mathrm{H}$. et al. Common and rare coding genetic variation underlying the electrocardiographic PR interval. Circ. Genom. Precis Med 11, e002037 (2018).

14. Genomes Project, C. et al. A global reference for human genetic variation. Nature 526, 68-74 (2015).

15. Liu, Y. et al. SPSB3 targets SNAIL for degradation in GSK-3beta phosphorylation-dependent manner and regulates metastasis. Oncogene 37, 768-776 (2018).

16. Holm, H. et al. A rare variant in MYH6 is associated with high risk of sick sinus syndrome. Nat. Genet 43, 316-20 (2011).

17. Thorolfsdottir, R. B. et al. A Missense Variant in PLEC Increases Risk of Atrial Fibrillation. J. Am. Coll. Cardiol. 70, 2157-2168 (2017).

18. Consortium, G. T. et al. Genetic effects on gene expression across human tissues. Nature 550, 204-213 (2017).

19. Schmitt, A. D. et al. A compendium of chromatin contact maps reveals spatially active regions in the human genome. Cell Rep. 17, 2042-2059 (2016).

20. Nielsen, J. B. et al. Genome-wide study of atrial fibrillation identifies seven risk loci and highlights biological pathways and regulatory elements involved in cardiac development. Am. J. Hum. Genet 102, 103-115 (2018).

21. Roselli, C. et al. Multi-ethnic genome-wide association study for atrial fibrillation. Nat. Genet. 50, 1225-1233 (2018).

22. Pers, T. H. et al. Biological interpretation of genome-wide association studies using predicted gene functions. Nat. Commun. 6, 5890 (2015).

23. Sudlow, C. et al. UK biobank: an open access resource for identifying the causes of a wide range of complex diseases of middle and old age. PLoS Med. 12, e1001779 (2015)
24. Bermudez-Jimenez, F. J. et al. Novel desmin mutation p.Glu401Asp impairs filament formation, disrupts cell membrane integrity, and causes severe arrhythmogenic left ventricular cardiomyopathy/dysplasia. Circulation 137, 1595-1610 (2018)

25. Norgett, E. E. et al. Recessive mutation in desmoplakin disrupts desmoplakinintermediate filament interactions and causes dilated cardiomyopathy, woolly hair and keratoderma. Hum. Mol. Genet. 9, 2761-6 (2000).

26. Rampazzo, A. et al. Mutation in human desmoplakin domain binding to plakoglobin causes a dominant form of arrhythmogenic right ventricular cardiomyopathy. Am. J. Hum. Genet. 71, 1200-6 (2002).

27. Taylor, M. R. et al. Prevalence of desmin mutations in dilated cardiomyopathy. Circulation 115, 1244-51 (2007).

28. van Tintelen, J. P. et al. Severe cardiac phenotype with right ventricular predominance in a large cohort of patients with a single missense mutation in the DES gene. Heart Rhythm 6, 1574-83 (2009).

29. Glukhov, A. V. et al. Conduction remodeling in human end-stage nonischemic left ventricular cardiomyopathy. Circulation 125, 1835-47 (2012).

30. Gomes, J. et al. Electrophysiological abnormalities precede overt structural changes in arrhythmogenic right ventricular cardiomyopathy due to mutations in desmoplakin-A combined murine and human study. Eur. Heart J. 33, 1942-53 (2012).

31. Fukuzawa, A. et al. Interactions with titin and myomesin target obscurin and obscurin-like 1 to the M-band: implications for hereditary myopathies. J. Cell Sci. 121, 1841-51 (2008).

32. Cheng, H. et al. Loss of enigma homolog protein results in dilated cardiomyopathy. Circ. Res 107, 348-56 (2010).

33. Hojayev, B., Rothermel, B. A., Gillette, T. G. \& Hill, J. A. FHL2 binds calcineurin and represses pathological cardiac growth. Mol. Cell Biol. 32, 4025-34 (2012).

34. Friedrich, F. W. et al. FHL2 expression and variants in hypertrophic cardiomyopathy. Basic Res. Cardiol. 109, 451 (2014).

35. Dierck, F. et al. The novel cardiac z-disc protein CEFIP regulates cardiomyocyte hypertrophy by modulating calcineurin signaling. J. Biol. Chem. 292, 15180-15191 (2017).

36. Duhme, N. et al. Altered $\mathrm{HCN} 4$ channel C-linker interaction is associated with familial tachycardia-bradycardia syndrome and atrial fibrillation. Eur. Heart J. 34, 2768-75 (2013)

37. Milanesi, R., Baruscotti, M., Gnecchi-Ruscone, T. \& DiFrancesco, D. Familial sinus bradycardia associated with a mutation in the cardiac pacemaker channel. N. Engl. J. Med 354, 151-7 (2006).

38. Milano, A. et al. HCN4 mutations in multiple families with bradycardia and left ventricular noncompaction cardiomyopathy. J. Am. Coll. Cardiol. 64, 745-56 (2014).

39. Priori, S. G. et al. Mutations in the cardiac ryanodine receptor gene (hRyR2) underlie catecholaminergic polymorphic ventricular tachycardia. Circulation 103, 196-200 (2001).

40. Kubo, T. et al. Cloning, sequencing and expression of complementary DNA encoding the muscarinic acetylcholine receptor. Nature 323, 411-6 (1986).

41. Kurachi, Y. G protein regulation of cardiac muscarinic potassium channel. Am. J. Physiol. 269, C821-30 (1995)

42. Aistrup, G. L. et al. Targeted G-protein inhibition as a novel approach to decrease vagal atrial fibrillation by selective parasympathetic attenuation. Cardiovasc. Res. 83, 481-92 (2009).

43. Dobrev, D. et al. Molecular basis of downregulation of G-protein-coupled inward rectifying $\mathrm{K}(+)$ current $(\mathrm{I}(\mathrm{K}, \mathrm{ACh})$ in chronic human atrial fibrillation: decrease in GIRK4 mRNA correlates with reduced I(K,ACh) and muscarinic receptor-mediated shortening of action potentials. Circulation 104, 2551-7 (2001).

44. Stavrakis, $\mathrm{S}$. et al. Activating autoantibodies to the beta- 1 adrenergic and $\mathrm{m} 2$ muscarinic receptors facilitate atrial fibrillation in patients with Graves' hyperthyroidism. J. Am. Coll. Cardiol. 54, 1309-16 (2009).

45. Winkler, T. W. et al. Quality control and conduct of genome-wide association meta-analyses. Nat. Protoc. 9, 1192-212 (2014).

46. Willer, C. J., Li, Y. \& Abecasis, G. R. METAL: fast and efficient meta-analysis of genomewide association scans. Bioinformatics 26, 2190-1 (2010).

47. Higgins, J. P., Thompson, S. G., Deeks, J. J. \& Altman, D. G. Measuring inconsistency in meta-analyses. BMJ 327, 557-60 (2003).

48. Pruim, R. J. et al. LocusZoom: regional visualization of genome-wide association scan results. Bioinformatics 26, 2336-7 (2010).

49. Yang, J., Lee, S. H., Goddard, M. E. \& Visscher, P. M. GCTA: a tool for genome-wide complex trait analysis. Am. J. Hum. Genet 88, 76-82 (2011).

50. Loh, P. R. et al. Contrasting genetic architectures of schizophrenia and other complex diseases using fast variance-components analysis. Nat. Genet. 47, 1385-92 (2015).

51. McLaren, W. et al. The ensembl variant effect predictor. Genome Biol. 17, 122 (2016). 
52. Kumar, P., Henikoff, S. \& Ng, P. C. Predicting the effects of coding nonsynonymous variants on protein function using the SIFT algorithm. Nat. Protoc. 4, 1073-81 (2009).

53. Adzhubei, I., Jordan, D. M. \& Sunyaev, S. R. Predicting functional effect of human missense mutations using PolyPhen-2. Curr Protoc Hum Genet Chapter 7, Unit7 20 (2013).

54. Bernstein, B. E. et al. The NIH roadmap epigenomics mapping consortium Nat. Biotechnol. 28, 1045-8 (2010).

55. Ward, L. D. \& Kellis, M. HaploReg: a resource for exploring chromatin states, conservation, and regulatory motif alterations within sets of genetically linked variants. Nucleic Acids Res. 40, D930-4 (2012).

56. Barbeira, A. N. et al. Exploring the phenotypic consequences of tissue specific gene expression variation inferred from GWAS summary statistics. Nat. Commun. 9, 1825 (2018)

57. Iotchkova, V. et al. GARFIELD classifies disease-relevant genomic features through integration of functional annotations with association signals. Nat. Genet. 51, 343-353 (2019).

58. Consortium, E. P. An integrated encyclopedia of DNA elements in the human genome. Nature 489, 57-74 (2012).

59. Buniello, A. et al. The NHGRI-EBI GWAS Catalog of published genome-wide association studies, targeted arrays and summary statistics 2019. Nucleic Acids Res 47, D1005-D1012 (2019).

60. Staley, J. R. et al. PhenoScanner: a database of human genotype-phenotype associations. Bioinformatics 32, 3207-3209 (2016).

61. Purcell, S. et al. PLINK: a tool set for whole-genome association and population-based linkage analyses. Am. J. Hum. Genet. 81, 559-75 (2007).

\section{Acknowledgements}

We provide all investigator and study-specific acknowledgements in Supplementary Note 1 , and funding sources in Supplementary Note 2.

\section{Author contributions}

Interpreted results, writing, and editing the manuscript: I.N., L.-C.W., S.A.L., and P.B.M. Conceptualization and supervision of project: S.A.L. and P.B.M. Contributed to GWAS analysis plan: I.N., L.-C.W., H.R.W., Y.J., S.A.L., and P.B.M. Performed meta-analyses: I.N. and L.-C.W. Performed GCTA, heritability, geneset enrichment, and pathway analyses, variant annotations: I.N. Performed PRS and gene expression analyses: S.H.C., M.D.C., and L.-C.W. Performed HiC analyses: I.N., M.R.B., B.M., and P.B.M. Performed gene literature review: I.N., L.-C.W., A.W.Hall, N.R.T., M.D.C., J.H.C., J.J.C., A.T., Y.J., S.A.L., and P.B.M. Contributed to study-specific GWAS by providing phenotype, genotype and performing data analyses: J.M., I.R., C.H., P.G., M.P.C., T.B., O.P., I.K., E.T., N.M.A., R.P.S., M.F.L., A.L.P.R., A.M., V.G., E.I., A.P.M., F.D.M., L.F., M.G., A.A.H., J.P. C., L.L., C.M.L., J.S., N.J.S., C.P.N., M.B.R., S.U., G.S., P.P.M., M.K., N.M., K.N., I.N., M.J. C., A.D., S.P., M.E.M., J.R.O., A.R.S., K.R., D.C., L.R., S.A., S.T., T.L., O.T.R., N.H., L.P.L, J.F.W., P.K.J., C.L.K.B., H.C., C.M.v., J.A.K., A.I., P.L.H., L.-C.W., S.A.L., P.T.E., T.B.H., L. J.L., A.V.S., V.G., E.P.B., R.J.F.L., G.N.N., M.H.P., A.C., H.M., J.W., M.M.-N., A.P., T.M., M.W., T.D.S., Y.J., M.M., M.R., Y.J.V., P.H., N.V., K.S., S.K., K.S., M.F.S., B.L., C.R., D.F., M.J.C., M.O., D.M.R., M.B.S., J.G.S, J.A.B., M.L.B., J.C.B., B.M.P., N.S., K.R., C.P., P.P.P., A.G., C.F., J.W.J., I.F., P.W.M., S.T., S.W., M.D., S.B.F., U.V., A.S.H., A.J., K.S., V.S., S.R. H., J.I.R., X.G., H.J.L., J.Y., K.D.T., R.N., R.d., D.O.M., A.C.M., F.C., J.D., E.G.L., Y.Q., K.V.T., E.J.B., D.L., H.L., C.H.N., K.L.L., A.D.M., D.J.P., B.H.S., B.H.S., M.E.v, A.U., J.H.,
R.D.J., U.P., A.P.R., E.A.W., C.K., E.B., D.E.A., G.B.E., A.A., E.Z.S., C.L.A., S.M.G., K.F.K., C.C.L., A.A.S., A.S., S.A., M.A.S., M.Y.v., P.D.L., A.T., M.O., J.R., S.V.D., P.B.M., K.S., H.H., P.S., G.S., G.T., R.B.T., U.T., D.O.A., D.F.G. All authors read, revised, and approved the manuscript.

\section{Conflict of interest}

I.N. became a full-time employee of Gilead Sciences Ltd following submission of the manuscript. S.A.L. receives sponsored research support from Bristol Myers Squibb/ Pfizer, Bayer AG, and Boehringer Ingelheim, and has consulted for Bristol Myers Squibb/Pfizer and Bayer AG. P.T.E. is supported by a grant from Bayer AG to the Broad Institute focused on the genetics and therapeutics of cardiovascular diseases. P. T.E. has also served on advisory boards or consulted for Bayer AG, Quest Diagnostics, and Novartis. M.J.C. is Chief Scientist for Genomics England, a UK Government company. B.M.P. serves on the DSMB of a clinical trial funded by Zoll LifeCor and on the Steering Committee of the Yale Open Data Access Project funded by Johnson \& Johnson. V.S. has participated in a conference trip sponsored by Novo Nordisk and received a modest honorarium for participating in an advisory board meeting. K.S. H.H., P.S., G.S., G.T., R.B.T., U.T., D.O.A., D.F.G. are employed by deCODE genetics/ Amgen Inc. E.I. is employed by GlaxoSmithKline. A.M. is employed by Genentech Inc.

\section{Additional information}

Supplementary information is available for this paper at https://doi.org/10.1038/s41467 020-15706-x.

Correspondence and requests for materials should be addressed to S.A.L. or P.B.M.

Peer review information Nature Communications thanks Jennifer Huffman, Norihiro Kato and Marco Perez for their contribution to the peer review of this work. Peer reviewer reports are available.

Reprints and permission information is available at http://www.nature.com/reprints

Publisher's note Springer Nature remains neutral with regard to jurisdictional claims in published maps and institutional affiliations.

\begin{abstract}
Open Access This article is licensed under a Creative Commons Attribution 4.0 International License, which permits use, sharing, adaptation, distribution and reproduction in any medium or format, as long as you give appropriate credit to the original author(s) and the source, provide a link to the Creative Commons license, and indicate if changes were made. The images or other third party material in this article are included in the article's Creative Commons license, unless indicated otherwise in a credit line to the material. If material is not included in the article's Creative Commons license and your intended use is not permitted by statutory regulation or exceeds the permitted use, you will need to obtain permission directly from the copyright holder. To view a copy of this license, visit http://creativecommons.org/ licenses/by/4.0/.
\end{abstract}

(C) The Author(s) 2020

loanna Ntalla1,145, Lu-Chen Weng (10) 2,3,145, James H. Cartwright ${ }^{1}$, Amelia Weber Hall (10) ${ }^{2,3}$,

Gardar Sveinbjornsson ${ }^{4}$, Nathan R. Tucker (10) ${ }^{2,3}$, Seung Hoan Choi ${ }^{3}$, Mark D. Chaffin (10 ${ }^{3}$, Carolina Roselli (10) ${ }^{3,5}$, Michael R. Barnes (10) 1,6, Borbala Mifsud (1) 1,7, Helen R. Warren ${ }^{1,6}$, Caroline Hayward (10) ${ }^{8}$, Jonathan Marten 8 , James J. Cranley ${ }^{1}$, Maria Pina Concas (1) ${ }^{9}$, Paolo Gasparini ${ }^{9,10}$, Thibaud Boutin (10) ${ }^{8}$, Ivana Kolcic (1) ${ }^{11}$, Ozren Polasek ${ }^{11,12,13}$, Igor Rudan (10 ${ }^{14}$, Nathalia M. Araujo ${ }^{15}$, Maria Fernanda Lima-Costa ${ }^{16}$, Antonio Luiz P. Ribeiro ${ }^{17}$, Renan P. Souza (10 ${ }^{15}$, Eduardo Tarazona-Santos ${ }^{15}$, Vilmantas Giedraitis (1) ${ }^{18}$, Erik Ingelsson (1) 19,20,21,22, Anubha Mahajan (1) ${ }^{23}$, Andrew P. Morris (1) 23,24,25, Fabiola Del Greco M ${ }^{26}$, Luisa Foco ${ }^{26}$, Martin Gögele ${ }^{26}$, Andrew A. Hicks (10 26, James P. Cook ${ }^{24}$, Lars Lind ${ }^{27}$, Cecilia M. Lindgren28,29,30, Johan Sundström (iD ${ }^{31}$, Christopher P. Nelson (10) ${ }^{32,33}$, Muhammad B. Riaz (D) ${ }^{32,33}$, Nilesh J. Samani ${ }^{32,33}$, 
Gianfranco Sinagra (1) ${ }^{34}$, Sheila Ulivi ${ }^{9}$, Mika Kähönen ${ }^{35,36}$, Pashupati P. Mishra (1) 37,38, Nina Mononen ${ }^{37,38}$, Kjell Nikus ${ }^{39,40}$, Mark J. Caulfield (1) 1,6, Anna Dominiczak (10) ${ }^{41}$, Sandosh Padmanabhan 41,42, May E. Montasser 43,44 , Jeff R. O'Connell ${ }^{43,44}$, Kathleen Ryan ${ }^{43,44}$, Alan R. Shuldiner 43,44 , Stefanie Aeschbacher ${ }^{45}$, David Conen 45,46, Lorenz Risch (1) 47,48,49, Sébastien Thériault ${ }^{46,50,}$ Nina Hutri-Kähönen ${ }^{51,52}$, Terho Lehtimäki ${ }^{37,38}$, Leo-Pekka Lyytikäinen 37,38,39, Olli T. Raitakari53,54,55, Catriona L. K. Barnes ${ }^{14}$, Harry Campbell ${ }^{14}$, Peter K. Joshi (1) ${ }^{14}$, James F. Wilson (1) ${ }^{8,14}$, Aaron Isaacs (ID ${ }^{56}$, Jan A. Kors ${ }^{57}$, Cornelia M. van Duijn (10 ${ }^{58}$, Paul L. Huang ${ }^{2}$, Vilmundur Gudnason (10 59,60, Tamara B. Harris ${ }^{61}$, Lenore J. Launer ${ }^{61}$, Albert V. Smith (10) 59,62, Erwin P. Bottinger ${ }^{63}$, Ruth J. F. Loos (1) 63,64, Girish N. Nadkarni63, Michael H. Preuss (1] ${ }^{63}$, Adolfo Correa ${ }^{65}$, Hao Mei ${ }^{66}$, James Wilson ${ }^{67}$, Thomas Meitinger ${ }^{68,69,70}$, Martina Müller-Nurasyid (1D 68,71,72,73, Annette Peters 68,74,75, Melanie Waldenberger (1) 68,75,76, Massimo Mangino (10) 77,78, Timothy D. Spector ${ }^{77}$, Michiel Rienstra (1D ${ }^{5}$, Yordi J. van de Vegte ${ }^{5}$, Pim van der Harst (10 ${ }^{5}$, Niek Verweij ${ }^{5,79}$, Stefan Kääb ${ }^{68,73}$, Katharina Schramm ${ }^{68,71,73}$, Moritz F. Sinner ${ }^{68,73}$, Konstantin Strauch 71,72 , Michael J. Cutler 80 , Diane Fatkin (10 81,82,83, Barry London ${ }^{84}$, Morten Olesen ${ }^{85,86,}$ Dan M. Roden ${ }^{87}$, M. Benjamin Shoemaker 88 , J. Gustav Smith ${ }^{89}$, Mary L. Biggs ${ }^{90,91}$, Joshua C. Bis 90 , Jennifer A. Brody (10 90, Bruce M. Psaty 90,92,93, Kenneth Rice (1) ${ }^{91}$, Nona Sotoodehnia90,92,94, Alessandro De Grandi ${ }^{26}$, Christian Fuchsberger ${ }^{26}$, Cristian Pattaro (1) ${ }^{26}$, Peter P. Pramstaller ${ }^{26}$, lan Ford ${ }^{95}$, J. Wouter Jukema (1) ${ }^{96,97}$, Peter W. Macfarlane ${ }^{98}$, Stella Trompet ${ }^{99}$, Marcus Dörr ${ }^{100,101}$, Stephan B. Felix ${ }^{100,101}$, Uwe Völker 100,102, Stefan Weiss (100,102, Aki S. Havulinna (103,104, Antti Jula103, Katri Sääksjärvi (103) Veikko Salomaa (103 ${ }^{103}$, Xiuqing Guo ${ }^{105}$, Susan R. Heckbert ${ }^{106}$, Henry J. Lin ${ }^{105}$, Jerome I. Rotter (iD ${ }^{105}$, Kent D. Taylor (105, Jie Yao ${ }^{107}$, Renée de Mutsert ${ }^{108}$, Arie C. Maan96, Dennis O. Mook-Kanamori ${ }^{108,109}$, Raymond Noordam 99 , Francesco Cucca ${ }^{110}$, Jun Ding ${ }^{111}$, Edward G. Lakatta (11) ${ }^{112}$, Yong Qian ${ }^{111}$, Kirill V. Tarasov ${ }^{112}$, Daniel Levy113,114, Honghuang Lin (10 114,115, Christopher H. Newton-Cheh3,116, Kathryn L. Lunetta (1) 114,117, Alison D. Murray (1) ${ }^{118}$, David J. Porteous (119,120, Blair H. Smith (D) ${ }^{121}$, Bruno H. Stricker ${ }^{122}$, André Uitterlinden (10 ${ }^{123}$, Marten E. van den Berg ${ }^{122}$, Jeffrey Haessler ${ }^{124}$, Rebecca D. Jackson ${ }^{125}$, Charles Kooperberg ${ }^{124}$, Ulrike Peters (1) ${ }^{124}$, Alexander P. Reiner ${ }^{124,126}$, Eric A. Whitsel ${ }^{127}$, Alvaro Alonso (1) ${ }^{128,}$ Dan E. Arking ${ }^{129}$, Eric Boerwinkle ${ }^{130}$, Georg B. Ehret (13) ${ }^{131}$, Elsayed Z. Soliman (10 ${ }^{132}$, Christy L. Avery ${ }^{133,134}$, Stephanie M. Gogarten (10 91, Kathleen F. Kerr (10) ${ }^{91}$, Cathy C. Laurie ${ }^{91}$, Amanda A. Seyerle ${ }^{135}$, Adrienne Stilp ${ }^{91}$, Solmaz Assa5, M. Abdullah Said (10 5 , M. Yldau van der Ende ${ }^{5}$, Pier D. Lambiase ${ }^{136,137}$, Michele Orini (10) ${ }^{136,138,}$ Julia Ramirez (1) 1,137, Stefan Van Duijvenboden (1,137, David O. Arnar 4,60,139, Daniel F. Gudbjartsson (1) 4,140,

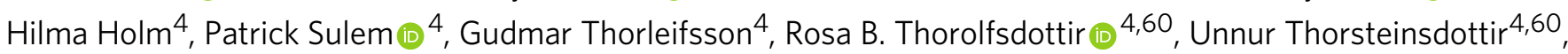
Emelia J. Benjamin (114,141,142, Andrew Tinker ${ }^{1,6}$, Kari Stefansson (10) 4,60, Patrick T. Ellinor (10) 2,3,143,

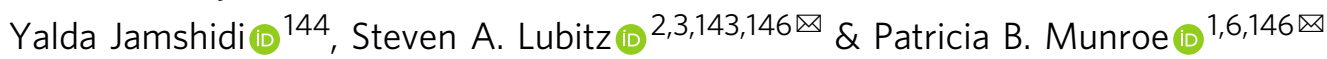

\footnotetext{
${ }^{1}$ William Harvey Research Institute, Barts and The London School of Medicine and Dentistry, Queen Mary University of London, London, UK. ${ }^{2}$ Cardiovascular Research Center, Massachusetts General Hospital, Boston, MA, USA. ${ }^{3}$ Program in Medical and Population Genetics, The Broad Institute of MIT and Harvard, Cambridge, MA, USA. ${ }^{4}$ deCODE genetics/Amgen, Inc., Reykjavik, Iceland. ${ }^{5}$ Department of Cardiology, University of Groningen, University Medical Center Groningen, Groningen, The Netherlands. ${ }^{6}$ National Institute for Health Research, Barts Cardiovascular Biomedical Research Centre, Queen Mary University of London, London, UK. ${ }^{7}$ College of Health and Life Sciences, Hamad Bin Khalifa University, Education City, Doha, Qatar. ${ }^{8}$ Medical Research Council Human Genetics Unit, Institute of Genetics and Molecular Medicine, University of Edinburgh, Edinburgh, UK. ${ }^{9}$ Institute for Maternal and Child Health-IRCCS 'Burlo Garofolo', Trieste, Italy. ${ }^{10}$ Department of Medicine, Surgery and Health Science, University of Trieste, Trieste, Italy. ${ }^{11}$ University of Split School of Medicine, Split, Croatia. ${ }^{12}$ Clinical Hospital Centre Split,

Split, Croatia. ${ }^{13}$ Psychiatric Hospital Sveti Ivan, Zagreb, Croatia. ${ }^{14}$ Centre for Global Health Research, Usher Institute of Population Health Sciences and Informatics, University of Edinburgh, Edinburgh, UK. ${ }^{15}$ Departamento de Biologia Geral, Universidade Federal de Minas Gerais, Belo Horizonte, Minas Gerais, Brazil. ${ }^{16}$ Rene Rachou Reserch Institute, Oswaldo Cruz Foundation, Belo Horizonte, Minas Gerais, Brazil. ${ }^{17}$ Hospital das Clínicas e Faculdade de Medicina, Universidade Federal de Minas Gerais, Belo Horizonte, Minas Gerais, Brazil. ${ }^{18}$ Department of Public Health, Geriatrics, Uppsala University, Uppsala, Sweden. ${ }^{19}$ Department of Medicine, Division of Cardiovascular Medicine, Stanford University School of Medicine, Stanford, CA, USA. ${ }^{20}$ Stanford Cardiovascular Institute, Stanford University, Stanford, CA, USA. ${ }^{21}$ Stanford Diabetes Research Center, Stanford University, Stanford, CA, USA. ${ }^{22}$ Department of Medical Sciences, Molecular Epidemiology and Science for Life Laboratory, Uppsala University, Uppsala, Sweden. ${ }^{23}$ Wellcome Centre for Human Genetics, University of Oxford, Oxford, UK. ${ }^{24}$ Department of Biostatistics, University of Liverpool, Liverpool, UK. ${ }^{25}$ Division of Musculoskeletal and Dermatological Sciences, University of Manchester, Manchester, UK. ${ }^{26}$ Institute for Biomedicine,
} 
Eurac Research, Affiliated Institute of the University of Lübeck, Bolzano, Italy. ${ }^{27}$ Medical Sciences, Uppsala University Hospital, Uppsala, Sweden. ${ }^{28}$ Nuffield Department of Medicine, Li Ka Shing Centre for Health Information and Discovery, Big Data Institute, University of Oxford, Oxford, UK. ${ }^{29}$ Nuffield Department of Medicine, The Wellcome Centre for Human Genetics, University of Oxford, Oxford, UK. ${ }^{30}$ The Broad Institute of MIT and Harvard, Cambridge, MA, USA. ${ }^{31}$ Department of Medical Sciences, Uppsala University, Uppsala, Sweden. ${ }^{32}$ Department of Cardiovascular Sciences, Cardiovascular Research Centre, Glenfield Hospital, University of Leicester, Leicester, UK. ${ }^{33}$ NIHR Leicester Biomedical Research Centre, Glenfield Hospital, Groby Road, Leicester, UK. ${ }^{34}$ Cardiovascular Department, Azienda Ospedaliera Universitaria Integrata of Trieste, Trieste, Italy. ${ }^{35}$ Department of Clinical Physiology, Tampere University Hospital, Tampere, Finland. ${ }^{36}$ Department of Clinical Physiology, Faculty of Medicine and Health Technology, Finnish Cardiovascular Research Center Tampere University, Tampere, Finland. ${ }^{37}$ Department of Clinical Chemistry, Fimlab Laboratories, Tampere, Finland. ${ }^{38}$ Department of Clinical Chemistry, Faculty of Medicine and Health Technology, Finnish Cardiovascular Research Center, Tampere University, Tampere, Finland. ${ }^{39}$ Department of Cardiology, Heart Center, Tampere University Hospital, Tampere, Finland. ${ }^{40}$ Department of Cardiology, Finnish Cardiovascular Research Center, Faculty of Medicine and Health Technology, Tampere University, Tampere, Finland. ${ }^{41}$ Institute of Cardiovascular and Medical Sciences, College of Medical, Veterinary and Life Sciences, University of Glasgow, Glasgow, UK. ${ }^{42}$ Institute of Cardiovascular and Medical Sciences, University of Glasgow, Glasgow, UK. ${ }^{43}$ Division of Endocrinology, Diabetes, and Nutrition, University of Maryland School of Medicine, Baltimore, MD, USA. ${ }^{44}$ Program for Personalized and Genomic Medicine, University of Maryland School of Medicine, Baltimore, MD, USA. ${ }^{45}$ Cardiology Division, University Hospital, Basel, Switzerland. ${ }^{46}$ Population Health Research Institute, McMaster University, Hamilton, ON, Canada. ${ }^{47}$ Institute of Clinical Chemistry, Inselspital Bern, University Hospital, University of Bern, Bern, Switzerland. ${ }^{48}$ Labormedizinisches Zentrum Dr. Risch, Vaduz, Liechtenstein. ${ }^{49}$ Private University of the Principality of Liechtenstein, Triesen, Liechtenstein. ${ }^{50}$ Department of Molecular Biology, Medical Biochemistry and Pathology, Laval University, Quebec, QC, Canada. ${ }^{51}$ Department of Pediatrics, Tampere University Hospital, Tampere, Finland. ${ }^{52}$ Department of Pediatrics, Faculty of Medicine and Health Technology, Tampere University, Tampere, Finland. ${ }^{53}$ Department of Clinical Physiology and Nuclear Medicine, Turku University Hospital, Turku, Finland. ${ }^{54}$ Research Centre of Applied and Preventive Cardiovascular Medicine, University of Turku, Turku, Finland. ${ }^{55}$ Centre for Population Health Research, University of Turku and Turku University Hospital, Turku, Finland. ${ }^{56}$ CARIM School for Cardiovascular Diseases, Maastricht Center for Systems Biology (MaCSBio), Department of Biochemistry, and Department of Physiology, Maastricht University, Maastricht, The Netherlands. ${ }^{57}$ Department of Medical Informatics Erasmus MC, University Medical Center Rotterdam, Rotterdam, The Netherlands. ${ }^{58}$ Genetic Epidemiology Unit, Department of Epidemiology, Erasmus University Medical Center, Rotterdam, The Netherlands. ${ }^{59}$ Icelandic Heart Association, Kopavogur, Iceland. ${ }^{60}$ Faculty of Medicine, University of Iceland, Reykjavik, Iceland. ${ }^{61}$ Laboratory of Epidemiology and Population Sciences, National Institute on Aging, NIH, Baltimore, MD, USA. ${ }^{62}$ School of Public Health, Department of Biostatistics, University of Michigan, Ann Arbor, MI, USA. ${ }^{63}$ The Charles Bronfman Institute for Personalized Medicine, Icahn School of Medicine at Mount Sinai, New York, NY, USA. ${ }^{64}$ The Mindich Child Health and Development Institute, Icahn School of Medicine at Mount Sinai, New York, NY, USA. ${ }^{65}$ Jackson Heart Study, Department of Medicine, University of Mississippi Medical Center, Jackson, MS, USA. ${ }^{66}$ Department of Data Science, University of Mississippi Medical Center, Jackson, MS, USA. ${ }^{67}$ Department of Physiology and Biophysics, University of Mississippi Medical Center, Jackson, MS, USA. ${ }^{68}$ DZHK (German Centre for Cardiovascular Research), Munich Heart Alliance, Munich, Germany. ${ }^{69}$ Institute of Human Genetics, Helmholtz Zentrum München - German Research Center for Environmental Health, Neuherberg, Germany. ${ }^{70}$ Institute of Human Genetics, Klinikum rechts der Isar, Technische Universität München, Munich, Germany. ${ }^{71}$ Institute of Genetic Epidemiology, Helmholtz Zentrum München - German Research Center for Environmental Health, Neuherberg, Germany. ${ }^{72}$ IBE, Faculty of Medicine, LMU Munich, Munich, Germany. ${ }^{73}$ Department of Internal Medicine I (Cardiology), Hospital of the Ludwig-Maximilians-University (LMU) Munich, Munich, Germany. ${ }^{74}$ German Center for Diabetes Research, Neuherberg, Germany. ${ }^{75}$ Institute of Epidemiology, Helmholtz Zentrum München-German Research Center for Environmental Health, Neuherberg, Germany. ${ }^{76}$ Research Unit of Molecular Epidemiology, Helmholtz Zentrum München-German Research Center for Environmental Health, Neuherberg, Germany. ${ }^{77}$ Department of Twin Research and Genetic Epidemiology, Kings College London, London, UK. ${ }^{78}$ NIHR Biomedical Research Centre at Guy's and St Thomas' Foundation Trust, London, UK. ${ }^{79}$ Genomics plc, Oxford, UK. ${ }^{80}$ Intermountain Heart Institute, Intermountain Medical Center, Murray, UT, USA. ${ }^{81}$ Molecular Cardiology and Biophysics Division, Victor Chang Cardiac Research Institute, Darlinghurst, NSW, Australia. ${ }^{82}$ Cardiology Department, St. Vincent's Hospital, Darlinghurst, NSW, Australia. ${ }^{3}$ St Vincent's Clinical School, Faculty of Medicine, UNSW Sydney, Kensington, NSW, Australia. ${ }^{84}$ Department of Cardiovascular Medicine, University of lowa, lowa City, IA, USA. ${ }^{85}$ Laboratory for Molecular Cardiology, Department of Cardiology, The Heart Centre, Rigshospitalet, University Hospital of Copenhagen, Copenhagen, Denmark. ${ }^{86}$ Department of Biomedical Sciences, University of Copenhagen, Copenhagen, Denmark. ${ }^{87}$ Departments of Medicine, Pharmacology, and Biomedical Informatics, Vanderbilt University Medical Center, Nashville, TN, USA. ${ }^{88}$ Department of Medicine, Vanderbilt University Medical Center, Nashville, TN, USA. ${ }^{89}$ Department of Cardiology, Clinical Sciences, Wallenberg Center for Molecular Medicine, Lund University Diabetes Center, Lund University and Skane University Hospital, Lund, Sweden. ${ }^{90}$ Cardiovascular Health Research Unit, Department of Medicine, University of Washington, Seattle, WA, USA. ${ }^{91}$ Department of Biostatistics, University of Washington, Seattle, WA, USA. ${ }^{92}$ Department of Epidemiology, University of Washington, Seattle, WA, USA. ${ }^{93}$ Kaiser Permanente Washington Health Research Institute, Seattle, WA, USA. ${ }^{94}$ Cardiology Division, University of Washington, Seattle, WA, USA. ${ }^{95}$ Robertson Center for Biostatistics, University of Glasgow, Glasgow, UK. ${ }^{96}$ Department of Cardiology, Leiden University Medical Center, Leiden, The Netherlands. ${ }^{97}$ Einthoven Laboratory for Experimental Vascular Medicine, Leiden University Medical Center, Leiden, The Netherlands. ${ }^{98}$ Institute of Health and Wellbeing, College of Medical, Veterinary and Life Sciences, University of Glasgow, Glasgow, UK. ${ }^{99}$ Department of Internal Medicine, section of Gerontology and Geriatrics, Leiden University Medical Center, Leiden, The Netherlands. ${ }^{100}$ DZHK (German Centre for Cardiovascular Research), Greifswald, Germany. ${ }^{101}$ Department of Internal Medicine B - Cardiology, Pneumology, Infectious Diseases, Intensive Care Medicine, University Medicine Greifswald, Greifswald, Germany. ${ }^{102}$ Interfaculty Institute for Genetics and Functional Genomics; Department of Functional Genomics; University Medicine and University of Greifswald, Greifswald, Germany. ${ }^{103}$ Finnish Institute for Health and Welfare, Helsinki, Finland. ${ }^{104}$ Institute for Molecular Medicine Finland (FIMM), HiLIFE, University of Helsinki, Helsinki, Finland. ${ }^{105}$ Institute for Translational Genomics and Population Sciences and Department of Pediatrics, Los Angeles Biomedical Research Institute at Harbor-UCLA Medical Center, Torrance, CA, USA. ${ }^{106}$ Cardiovascular Health Research Unit and Department of Epidemiology, University of Washington, Seattle, WA, USA. ${ }^{107}$ Institute for Translational Genomics and Population Sciences, Los Angeles Biomedical Research Institute at Harbor-UCLA Medical Center, Torrance, CA, USA. ${ }^{108}$ Department of Clinical Epidemiology, Leiden University Medical Center, Leiden, The Netherlands. ${ }^{109}$ Department of Public Health and Primary Care, Leiden University Medical Center, Leiden, The Netherlands. ${ }^{110}$ Department of Biomedical Sciences, University of Sassari, Sassari, Italy. ${ }^{111}$ Laboratory of Genetics and Genomics, NIA/NIH, Baltimore, MD, USA. ${ }^{112}$ Laboratory of Cardiovascular Science, NIA/NIH, Baltimore, MD, USA. ${ }^{113}$ Population Sciences Branch, Division of Intramural Research, National Heart, Lung, and Blood Institute, Bethesda, MD, USA. ${ }^{114}$ National Heart Lung and Blood Institute's and Boston University's Framingham Heart Study, Framingham, MA, USA. ${ }^{115}$ Section of Computational Biomedicine, Department of Medicine, Boston University School of Medicine, Boston, MA, USA. ${ }^{116}$ Center for Human Genetic Research and Cardiovascular Research Center, Harvard Medical School and Massachusetts General Hospital, Boston, MA, USA. ${ }^{117}$ Department of Biostatistics, Boston University School of Public Health, Boston, MA, USA. ${ }^{118}$ The Institute of Medical 
Sciences, Aberdeen Biomedical Imaging Centre, University of Aberdeen, Aberdeen, UK. ${ }^{119}$ Centre for Genomic and Experimental Medicine, Institute of Genetics \& Molecular Medicine, University of Edinburgh, Western General Hospital, Edinburgh, UK. ${ }^{120}$ Centre for Cognitive Ageing and Cognitive Epidemiology, University of Edinburgh, Edinburgh, UK. ${ }^{121}$ Division of Population Health and Genomics, Ninewells Hospital and Medical School, University of Dundee, Dundee, UK. ${ }^{122}$ Department of Epidemiology Erasmus MC, University Medical Center Rotterdam, Rotterdam, The Netherlands. ${ }^{123}$ Human Genotyping Facility Erasmus MC University Medical Center Rotterdam, Rotterdam, The Netherlands. ${ }^{124}$ Fred Hutchinson Cancer Research Center, Division of Public Health Sciences, Seattle, WA, USA. ${ }^{125}$ Division of Endocrinology, Diabetes and Metabolism, Ohio State University, Columbus, OH, USA. ${ }^{126}$ Department of Epidemiology, University of Washington, Seattle, WA, USA. ${ }^{127}$ Departments of Epidemiology and Medicine, Gillings School of Global Public Health and School of Medicine, University of North Carolina, Chapel Hill, NC, USA. ${ }^{128}$ Department of Epidemiology, Rollins School of Public Health, Emory University, Atlanta, GA, USA. ${ }^{129}$ McKusick-Nathans Institute of Genetic Medicine, Johns Hopkins University School of Medicine, Baltimore, MD, USA. ${ }^{130}$ Human Genetics Center, University of Texas Health Science Center at Houston, Houston, TX, USA. ${ }^{131}$ Cardiology, Geneva University Hospitals, Geneva, Switzerland. ${ }^{132}$ Epidemiological Cardiology Research Center, Wake Forest School of Medicine, Winston-Salem, NC, USA. ${ }^{133}$ Department of Epidemiology, University of North Carolina, Chapel Hill, NC, USA. ${ }^{134}$ Carolina Population Center, University of North Carolina, Chapel Hill, NC, USA. ${ }^{135}$ Division of Pharmaceutical Outcomes and Policy, University of North Carolina, Chapel Hill, NC, USA. ${ }^{136}$ Barts Heart Centre, St Bartholomews Hospital, London, UK. ${ }^{137}$ Institute of Cardiovascular Science, University College London, London, UK. ${ }^{138}$ Department of Mechanical Engineering, University College London, London, UK. ${ }^{139}$ Department of Medicine, Landspitali University Hospital, Reykjavik, Iceland. ${ }^{140}$ School of Engineering and Natural Sciences, University of Iceland, Reykjavik, Iceland.

${ }^{141}$ Section of Cardiovascular Medicine and Section of Preventive Medicine, Department of Medicine, Boston University School of Medicine, Boston, MA, USA. ${ }^{142}$ Department of Epidemiology, Boston University School of Public Health, Boston, MA, USA. ${ }^{143}$ Cardiac Arrhythmia Service, Massachusetts General Hospital, Boston, MA, USA. ${ }^{144}$ Genetics Research Centre, Molecular and Clinical Sciences Institute, St George's, University of London, London, UK. ${ }^{145}$ These authors contributed equally: Ioanna Ntalla, Lu-Chen Weng. ${ }^{146}$ These authors jointly supervised this work: Steven A. Lubitz, Patricia B. Munroe. ${ }^{凶}$ email: slubitz@mgh.harvard.edu; p.b.munroe@qmul.ac.uk 